\title{
Subcontratación en el Sector Forestal Maderero Chileno e Impacto del Manejo Forestal Sustentable Implementado en Empresa Mandante sobre el Clima Organizacional. Un Estudio de Caso
}

\author{
SUBCONTRACTING IN THE FORESTRY AND TIMBER INDUSTRY OF CHILE AND THE IMPACT OF THE SUSTAINABLE \\ FORESTRY MANAGEMENT IMPLEMENTED IN THE PRINCIPAL COMPANY OVER THE ORGANIZATIONAL CLIMATE. A \\ CASE STUDY
}

\author{
Luis Améstica-Rivas', César Moya Lara², Mauricio Salazar Botello³, Álvaro Acuña Hormazábal ${ }^{4}$ \\ 1. 3. 4. Académico Depto. Gestión Empresarial, Facultad de Ciencias Empresariales, Universidad del Bío-Bío, Chillán, Chile. \\ 2. Académico Depto. Ingeniería en Madera, Facultad de Ingeniería, Universidad del Bío-Bío, Concepción, Chile.
}

\begin{abstract}
RESUMEN
Las exigencias en la industria forestal están enfocadas a alcanzar estándares de productividad y un desempeño socialmente responsable con las comunidades, trabajadores y empresas contratistas. Al igual que en otros sectores, ha habido una importante especialización funcional que ha llevado a las grandes empresas a externalizar parte de sus procesos de la cadena productiva, generando importantes vínculos con empresas contratistas, quienes deben cumplir nuevos estándares internacionales. Es por ello que estas últimas deben preocuparse de generar las condiciones adecuadas para sus trabajadores y su productividad, donde la implementación de un estándar de manejo forestal sustentable podría incidir en cambios organizacionales en una empresa. Esta investigación recoge el caso de una empresa contratista de servicios forestales que concentra sus operaciones en la Región del Bío-Bío en Chile, aplicando una herramienta de diagnóstico de clima organizacional mediante encuestas al personal de faena, la que mide las percepciones que tienen los individuos de su lugar de trabajo bajo el enfoque de Koys y Decottis (1991) adaptado en la investigación realizada por Chiang et al. (2010). Con un Alpha de Cronbach de 0,80, destaca que la percepción de los encuestados en promedio como bueno (De acuerdo) y se ubica en 3,86 puntos. A su vez, se evidencia un número importante de respuestas, lo cual abre un debate sobre los factores consultados. Por tanto, el clima organizacional constituye una más de las dimensiones que está afectando los procesos de certificación, especialmente la incidencia de las empresas contratistas sobre las mandantes.
\end{abstract}

(Améstica-Rivas L, Moya C, Salazar M, Acuña A, 2016. Subcontratación en el Sector Forestal Maderero Chileno e Impacto del Manejo Forestal Sustentable Implementado en Empresa Mandante sobre el Clima Organizacional. Un Estudio de Caso. Cienc Trab. Ene-Abr; 18 [55]: 1-8).

Palabras claves: SECTOR FORESTAL, CLIMA ORGANIZACIONAL, MANEJO FORESTAL SUSTENTABLE, EXTERNALIZACIÓN, CHILE.

\section{ABSTRACT}

The requirements in the forestry industry are focused on reaching productivity standards and a socially responsible performance towards communities, workers and subcontracting companies. Just as in other sectors, an important functional specialization has occurred, and this has forced the big companies to outsource parts of their production chain processes, generating important links with subcontracting companies, which must meet new international standards. That is the reason why the latter must be concerned about generating the suitable conditions for their workers and their productivity, where the implementation of a sustainable forestry management standard could have an impact on the organizational changes in a company. This investigation takes the case of a forestry services subcontracting company that concentrates its operations in the Bio-Bio region in Chile, using an organizational environment evaluation tool through surveys answered by workers that measures the perceptions that individuals have about their working place under the Koys and Decottis (1991) approach, adapted in the investigation made by Chiang et al. (2010). With Cronbach's alpha of 0,80 , highlights that the average perception of the survey respondents was good (Agrees) and it is placed at 3,86 points. At the same time, an important number of "I am not sure" answers are shown which opens the debate around the asked factors. Therefore, the organizational environment constitutes one of the dimensions that are affecting the certification processes, specially the impact of the contracting companies on the principal companies.

Key words: FORESTRY SECTOR, ORGANIZATIONAL ENVIRONMENT, SUSTAINABLE FORESTRY MANAGEMENT, OUTSOURCING, CHILE.
Correspondencia / Correspondence:

Luis Améstica-Rivas

Avda. Andrés Bello s/n, Casilla 447

Concepción, Chile

Tel.: 56422463344

e-mail: lamestica@ubiobio.cl

Recibido: 24 de Noviembre de 2015 / Aceptado: 11 de Enero de 2016

\section{INTRODUCCIÓN}

En Chile, la madera constituye el segundo producto de exportación más importante después de los minerales. ${ }^{1}$ La expansión es atribuible en gran medida a la política forestal generada y, más concretamente, al Decreto Ley 701, aprobado por el gobierno militar en 1975 que ha proporcionado importantes subvenciones ${ }^{2}$, haciendo que las plantaciones industriales de árboles más rentable 
mediante la privatización de grandes franjas de terrenos públicos e impuestos, eliminando tanto en la tierra y los productos procedentes de estas áreas. ${ }^{3}$ Esta normativa, además de incentivar las plantaciones forestales, regula la utilización de todo tipo de bosques, la cual ha tenido un decisivo efecto sobre el incremento de la superficie de plantaciones en el país y en el consecuente desarrollo de la industria forestal derivada de ellas. ${ }^{1}$

Actualmente, existen importantes grupos económicos que concentran las más grandes empresas forestales del país, donde subsisten empresas de servicios de menor tamaño a su alrededor, las cuales se han constituido en un subsector clave para el dinamismo y competitividad del sector silvomaderero, con una generación de 122 mil empleos 4 , la cual ha ido con tendencia a la baja debido a la mecanización de sus procesos y aun así es una fuente importante de trabajo para el país. Dado que el sector está orientado principalmente hacia el mercado exportador, ello ha significado adecuarse rápidamente a exigencias internacionales y a nuevos cambios para mantener altos niveles de productividad. Dada las exigencias internacionales aplicadas a las forestales, las empresas de servicios de estas grandes entidades mandantes han tendido a incorporar innovaciones tecnológicas, capacitación especializada y mejora de las condiciones laborales de sus trabajadores. En este mismo sentido, Ackerknecht ${ }^{5}$ ha señalado que en la generación de lugares de trabajo más seguros y saludables han contribuido los códigos de buenas prácticas forestales como la aplicación de modelos de manejo forestal sostenible (MFS).

La certificación de manejo forestal sostenible (MFS) por parte de las empresas se ha desarrollado con distintos estándares existentes a nivel mundial, siendo las certificaciones más importantes: FSC (Forest Stwardship Council), CSA (Canadian Standars Association), SFI (Sustainable Forestry Initiative), ATFS (American Tree Farm System), TFT (Tropical Forest Trust), MTCC (National Timber Certification Council) y PEFC (Program for the Endorsement of Forest Certification); esta última norma, consolidada en Europa, es la base de homologación de la norma chilena CERTFOR (Sistema Chileno de Certificación de Manejo Forestal Sustentable) y que ha sido también adaptada en otros países de la región, como Brasil. ${ }^{6}$ En el país, la mayoría de las grandes empresas forestales chilenas mantienen certificaciones paralelas bajo dos estándares (FSC y CERTFOR). ${ }^{7,8}$ Dichas normas monitorean diversos aspectos del manejo y producción forestal, promoviendo la adecuación de prácticas productivas, ambientales y sociales en concordancia con las exigencias que imponen los mercados internacionales.

La certificación forestal internacional del Forest Stewardship CouncilTM (FSCTM) consta de diez principios y cincuenta y seis criterios, diseñados, principalmente, con referencia a los bosques manejados para la producción de madera y las plantaciones forestales. Dicho sistema de certificación provee un sello de legitimidad demandado en los mercados internacionales. La certificación forestal, bajo el estándar del Forest Stwardship Council (FSC) surge a principios del año 1990 como una alternativa de promover el manejo responsable de los bosques a nivel mundial. Esta iniciativa se produce luego del fracaso de experiencias anteriores, que promovían castigos económicos a empresas que no cumplían con una gestión sustentable de sus bosques. ${ }^{8}$

Uno de los principios, tanto de FSC como CERTFOR, tiene que ver con los derechos de los trabajadores, siendo el foco central mantener o elevar el bienestar social y económico, a largo plazo, de los trabajadores forestales. ${ }^{7}$ Estas normas son utilizadas en todo el mundo, no sólo exigible a las grandes empresas mandantes sino también a las empresas contratistas (outsourcing), con el fin de integrar las funciones sociales, ambientales y económicas, de manera que logren mantenerse en los mercados actuales o acceder a nuevos que demandan productos certificados. Es decir, el entorno es el que ha comenzado a exigir a las empresas, cualquiera sea esta, que incluya la dimensión social y ecológica, que son complementarias a la dimensión económica.

Otro de los principios en que se sustenta el manejo forestal sostenible (MFS) tiene que ver con las relaciones con las comunidades locales y los derechos de los trabajadores; las empresas forestales operan en un entorno en que hay seres humanos que viven en comunidades locales aledañas o que son trabajadores de las empresas. El buen manejo forestal incluye el respeto de los derechos de comunidades y trabajadores y no sólo eso, sino que tener una conducta y un rendimiento alto por el mejoramiento de las condiciones de las personas. ${ }^{7}$ Las empresas forestales que hacen buen manejo, además tratan de que las comunidades aledañas y los trabajadores progresen, que reciban entrenamiento, les apoyan en sus iniciativas, capacitan a sus trabajadores, les proveen los equipos de protección personal que la OIT recomienda por cada actividad forestal, escuchan sus legítimos reclamos y, por lo tanto, contribuyen proactivamente a la paz social. ${ }^{9}$ Es así como el principio 7 de CERTFOR establece que los responsables del manejo forestal deberán respetar los derechos directos e indirectos de los trabajadores forestales, compensarlos adecuada y equitativamente, salvaguardando su salud y seguridad en el trabajo.

En este contexto, las empresas forestales requieren de instrumentos que les permitan valorar la gestión social con el fin de retroalimentar el sistema, para una evaluación adecuada de las prácticas de responsabilidad social empresarial, cumplir estándares de certificación de productos, procesos y el monitoreo del desempeño general de la empresa. Y, sin duda, un elemento que es clave en las empresas de servicios forestales es el ámbito de los recursos humanos. Por lo tanto, la evaluación del clima organizacional es una condición básica, en términos de contar con ambientes laborales seguros, productivos y ambientalmente responsables y así cumplir con los requisitos relacionados a la salud, el trabajo y la seguridad, contemplados tanto en la certificación FSC y CERTFOR, indistintamente. ${ }^{5}$

Cada día se está más consciente de que la certificación es una opción de sobrevivencia que tienen las empresas forestales para acceder a nuevos mercados. Asimismo, conocer el clima organizacional es fundamental para la empresa ya que afecta el rendimiento del trabajador y del equipo. No sólo se refiere a la productividad sino también a la mejor utilización de las habilidades de los trabajadores, las cuales muchas veces se ven perjudicadas cuando el lugar de trabajo posee un clima hostil y desagradable. Esta investigación utilizó un instrumento que pretende medir las variables del clima organizacional de una empresa contratista del sector forestal maderero, constituyéndose en un trabajo piloto aplicado a este subsector, el cual se puede replicar a otras empresas contratistas que deben someterse a las exigencias de los procesos de certificación de las empresas mandantes. En una primera etapa se realizó la construcción del marco teórico, con la descripción del sector destacando el tema de la certificación, el fenómeno de la subcontratación y el clima organizacional, sustento metodológico del estudio. En la segunda parte, se procedió a la aplicación de una escala de medición para clima organizacional de Koys \& Decottis adaptada por Chiang, Salazar, Huerta \& Nuñez ${ }^{10}$, con los resultados, discusión y conclusión final. 


\section{Sector Forestal Maderero y la certificación de manejo forestal sostenible}

El sector forestal en Chile ha llegado a constituirse en uno de los sectores más dinamizadores de la economía chilena, que encuentra su explicación en el estímulo estatal a la privatización y exportación, sumado a un mercado mundial en expansión. ${ }^{11}$ Las exportaciones forestales han ido en aumento, creciendo sobre un 10\% promedio al año entre 2003 y 2013, y se han mantenido en torno al 7\% del total nacional. ${ }^{12}$ Las plantaciones forestales no sólo han contribuido al bienestar socioeconómico de Chile, sino que también han permitido significativos avances ambientales, destacándose la reducción de la presión sobre los bosques nativos y de la erosión. Además, es el segundo rubro de mayor importancia en las exportaciones después de la gran minería1 y cuya fortaleza se explica por la explotación de recursos naturales renovables, lo cual asegura su desarrollo sostenible y sustentable en el tiempo. ${ }^{13}$ El sector forestal puede dividirse en tres subsectores, el de la extracción de trozas o cosecha, el de la industria maderera y el de la industria del papel y la celulosa. ${ }^{14}$

En Chile existen 15,7 millones de hectáreas de bosques, de las cuales el 85\% corresponde a bosques nativos (13,3 millones de hectáreas) y un 15\% a plantaciones forestales (2,4 millones de hectáreas). ${ }^{12}$ Las plantaciones forestales han experimentado un acelerado desarrollo y son hoy la base de una fuerte industria forestal, compuesta en un $60 \%$ por pino radiata y un $40 \%$ por otras especies, destacando entre estas aquellas de eucaliptos que hoy alcanzan al 30\% de las plantaciones totales, con más de 700 mil hectáreas. ${ }^{1}$ En tanto, se le atribuye factor determinante del aumento de ingresos de las zonas rurales, ofreciendo nuevas fuentes de empleo local directa e indirectamente para los habitantes. ${ }^{3}$ Asimismo, la incorporación de terrenos erosionados a la actividad forestal permite, por un lado, disminuir la erosión de los suelos como también darle una posibilidad de desarrollo a los pequeños propietarios y tener una mayor disponibilidad de biomasa y productos asociados, lo que da la posibilidad de ofrecer a las pequeñas y medianas empresas forestales materia prima disponible para sus actividades productivas. ${ }^{13} \mathrm{El}$ aumento global en el consumo de productos forestales, como son la pulpa, papel, combustibles de madera y madera colocan a la industria forestal a un sector sensible con el medio ambiente en un papel fundamental a través de su dependencia e impactos en distintos servicios de los ecosistemas. ${ }^{15}$

A pesar de los importantes impactos económicos, algunos plantean que una de las mayores contradicciones del sector forestal chileno es que mientras ha habido una destacada expansión de las exportaciones forestales a partir de plantaciones de pino y eucalipto, principalmente distribuidas entre las regiones séptima a novena, la población de esas regiones no ha visto un real impacto en su calidad de vida ${ }^{13}$, sumado a los costos medioambientales y sociales en el territorio. Es así como Andersson et al. ${ }^{3}$ citan varios estudios, donde se evidencian temas atribuibles a las plantaciones forestales, como son: la disminución de recursos hídricos, disminución de la biodiversidad, aumento del uso de pesticidas, degradado de la infraestructura pública, el aumento de la pobreza, y la despoblación rápida de las zonas rurales. Al mismo tiempo, hace referencia a la correlación existente con las tasas de pobreza rural, dado que disminuyen las oportunidades de crecimiento de la economía. Ahora bien, con todas las externalidades que la actividad forestal genera, se le ha considerado como un estímulo para el dinamismo económico de las zonas rurales, ya que además de la creciente migración rural-urbana, muchas veces la calidad de los suelos donde se realiza la actividad silvícola no siempre permite oportunidades a las actividades agrícolas. ${ }^{12}$

Ahora bien, existe una amplia y heterogeneidad de realidades del sector forestal a lo largo de Chile y es en la Región del Bío-Bío donde se desarrolla en mayor escala la industria forestal ${ }^{16}$ siendo, a su vez, la región más tradicional con respecto a esta industria debido a que las principales plantas de celulosa, catalizadoras de la expansión forestal, fueron instaladas en su territorio. En 1990, la producción maderera en la región superaba apenas el 20\%, pero para el año 2012, esta cifra llegaba al 56,6\% ${ }^{14}$. A su vez, la Región del Bío-Bío ha tendido a mayor diversificación, donde las antiguas plantaciones de pino han sido progresivamente reemplazadas por eucalipto, con mayor rapidez que en el resto del país. Esta diferencia con el nivel nacional se debe, probablemente, a que la expansión hacia otras regiones se haya basado en producción de bajo costo, es decir, de pino radiata. ${ }^{14}$

Este escenario se ve reafirmado por la importancia que progresivamente han cobrado los sistemas de certificación forestal, que contemplan de forma preponderante la revisión permanente de las buenas prácticas forestales, que suponen cuidar las condiciones y mecanismos establecidos según estándares internacionales al cumplimiento de verificadores tanto en materias ambientales, económicos como sociales. Para lograr aquello, se utiliza como herramienta de certificación -en Chile como en Sudamérica- el sistema FSC y $\mathrm{PEFC}^{6}$, este último en su versión adaptada nacionalmente como CERTFOR, siendo el primero el de mayor presencia a nivel mundial, el cual contempla diez principios y criterios $^{8}$, como son: respeto de las leyes y tratados internacionales, derecho y responsabilidad de tenencia y uso de la tierra, respeto de los derechos de las comunidades indigenas, relaciones con las comunidades y derechos de los trabajadores, beneficios del bosque, estudio de impacto ambiental, plan de manejo, monitoreo y evaluación, mantenimiento de los bosques de alto valor de conservación y plantaciones de árboles. ${ }^{9}$

En Chile, el 70\% de las plantaciones del país han sido certificados con los sellos CERTFOR y/o FSC, mientras el promedio a nivel mundial es de solo el 30\%. ${ }^{12}$ La incorporación de la certificación del manejo forestal sostenible es un objetivo prioritario, ya que produce un provecho tangible para las comunidades locales, fomenta la sostenibilidad de los medios de vida, salvaguarda la biodiversidad de los ecosistemas, y contribuye a combatir el cambio climático debido a la deforestación que ha logrado evitar y a las emisiones por deforestación y degradación de los bosques. ${ }^{17} \mathrm{Al}$ mismo tiempo, el creciente interés público y la conciencia global de los problemas ambientales y sociales han intensificado las presiones sobre las empresas forestales en sus esfuerzos para equilibrar eficazmente demandas de las partes interesadas, de lo que se ha establecido como una inversión socialmente responsable ${ }^{18}$, donde la industria forestal está fuertemente enfocada en cuestiones ambientales con la adopción de la norma de orientación ISO 26000 de responsabilidad social. ${ }^{15}$

Ahora bien, en este enclave productivo que rodea al sector forestal, las empresas demandantes de los servicios forestales se caracterizan por ser los principales inversores y dado que están orientadas al mercado externo, se encuentran certificadas por algún sello de calidad. Por ello, es imperativa su preocupación por el desarrollo de las empresas de servicio que son sus subcontratistas, que aunque sean externos son activos partícipes de la cadena de producción forestal, traspasando a estas últimas los estándares en cuanto a condiciones 
laborales de los trabajadores y controlar rigurosamente que se cumplan. Más aun, cuando las condiciones de lejanía y aislamiento en que se desarrolla la actividad, el bajo nivel de escolarización de los trabajadores en general y el alto riesgo en accidentes, son una realidad de preocupación mundial. $\mathrm{Y}$ tal como se ha evidenciado anteriormente, el FSC y PEFC en su versión a la norma chilena CERTFOR, establece normas de prevención de riesgos en beneficio de los trabajadores. ${ }^{19}$

\section{Externalización de servicios forestales y condiciones laborales}

El nivel de empleo asociado al sector forestal ha sido relativamente estable entre los años 2010 - 2012, alcanzando el empleo directo a los 120 mil trabajadores y al sumar el empleo directo (en el mismo sector) con el indirecto (empleo en otros sectores relacionado a través de encadenamientos con el sector forestal) se alcanza unos 300 mil trabajadores. ${ }^{12}$

Actualmente, la externalización de funciones, llamada indistintamente tercerización, subcontratación u "outsourcing", es un fenómeno que se ha generalizado en Chile en los últimos años y presenta un aumento importante, con cifras superiores al cincuenta por ciento de las empresas que actualmente desarrollan sus funciones dentro del territorio nacional, inclusive se ha extendido hasta la esfera de las instituciones públicas ${ }^{20}$, siendo la Ley $\mathrm{N}^{\circ} 20.123$ la que establece normas a la subcontratación laboral como al suministro de trabajo, regulando las relaciones laborales y los derechos de los trabajadores; sin perjuicio, persisten ciertas carencias referidas a las faltas de garantías de los derechos colectivos de los trabajadores. ${ }^{21}$

En general, la externalización supone que una organización decide entregar a un tercero externo a ella la responsabilidad de cumplir con una etapa, proceso o tarea que compone la cadena de valor, y que no es parte del denominado corazón del negocio. ${ }^{20}$ Es decir, la externalización lleva a la organización a la contratación de nuevos proveedores y nuevas formas de asegurar la entrega de materias primas, componentes y servicios, en una relación convenida cuyo objetivo es el abastecimiento de un producto o servicio determinado y que permita generar un valor agregado para ambos.

Ahora bien, la externalización laboral, como manifestación de la descentralización productiva, comprende tanto a la subcontratación como al suministro de trabajo ${ }^{21}$; la externalización de funciones no sólo ha generado beneficios, gracias a la especialización de funciones y economías que se generan a lo largo de la cadena de valor, también han surgido algunos efectos indeseados, y, tal como señala Ganga ${ }^{20}$ para Chile, los menos beneficiados con la externalización han sido los trabajadores de las empresas contratistas, a raiz de lo cual ha sido necesario regular estas situaciones a través de la ley de subcontratación y trabajo transitorio vigente desde el año 2007, especialmente por la existencia de vacíos legales que algunas empresas han utilizado con el fin de disminuir costos, gracias a la flexibilización laboral y desregulación, en desmedro de los derechos y beneficios de los trabajadores. Asimismo, la existencia de regímenes laborales diversos que se aplican al interior de una misma empresa -uno definido para los trabajadores de la empresa usuaria y otro para los trabajadores suministrados- generalmente es más desventajoso para estos últimos. ${ }^{21}$

El sector forestal -y específicamente la forestación- ha desarrollado formas organizativas del trabajo basadas en la tercerización de las diferentes labores del proceso de producción ${ }^{22}$, donde los trabajadores subcontratados están presentes en toda la cadena productiva.
Más aun, según estudios de organismos internacionales, del total de trabajadores insertos en el sector primario, una parte minoritaria corresponde a trabajadores propios, que están ubicados principalmente en las plantas o fábricas. ${ }^{16}$ Asimismo, el carácter estacional de gran parte de las actividades agropecuarias genera en el sector una fuerte demanda de mano de obra y/o maquinaria en períodos acotados y, para hacer frente a estas necesidades, las empresas han implementado la tercerización de maquinaria y del factor trabajo, como estrategia desarrollada para la maximización de utilidades. ${ }^{22}$ Es creciente la separación de las actividades de servicios generales de las actividades específicas, extractivas o productivas en el sector primario y secundario o de servicios especializados en el sector secundario, por ejemplo la cosecha forestal, son encargadas a terceros por las grandes empresas. ${ }^{23}$

El sector forestal también crea valor agregado indirectamente al relacionarse con otros sectores, generando encadenamientos hacia atrás y adelante por sobre el promedio de la economía nacional. ${ }^{12}$ Al mismo tiempo, los cambios tecnológicos y organizativos en las empresas forestales han generado distintas formas de operar en la externalización, dejando en evidencia temas relativos a la seguridad en el trabajo y riesgos de accidentes y que se acentúan en la medida que exista un empleo precario, dada la exposición a altas cargas de trabajo y peligros para la salud humana. ${ }^{24}$ La externalización de trabajadores ciertamente es una alternativa para reducir costos, especialmente los trabajadores tercerizados que operan en la cosecha de tipo manual, aunque de igual forma la tercerización está presente desde la siembra hasta la cosecha, es decir, pasando por todas las etapas de la gestión forestal. A su vez, la selección de contratistas de servicios forestales está fuertemente mediatizada por relaciones de confianza y fidelidad con la empresa principal, la que muchas veces no implementa un proceso objetivo e imparcial de selección de contratistas ${ }^{23}$, lo que acentúa la competencia y precariedad del subsector.

En las investigaciones de Echeverria $^{23}$ se cita a López (2007), donde se establece que la subcontratación de faenas forestales es una práctica habitual. Es así como han proliferado en la Región del Bío-Bío cientos de empresas de servicios que operan para las grandes empresas forestales en las faenas de silvicultura, cosecha, aserradero, procesamiento industrial y celulosa. En el mismo estudio se establece que al año 2007, en virtud de disposiciones de la Ley 20.120 sobre subcontratación laboral, los primeros meses se efectuaron 2.491 certificaciones de contratistas de la actividad forestal en la región; entre ellas 1.019 empresas de servicios del sector silvícola, 555 del rubro cosecha forestal, 256 de la industria y 661 de sector transporte; estos últimos, por temas de indefinición de lo que es un trabajador forestal muchas veces son excluidos de las estadísticas del sector. ${ }^{23}$

Lo que es evidente, y tal como lo señala Echeverría ${ }^{23}$, la subcontratación ha penetrado en las actividades de apoyo a la producción y en las faenas directas de producción, haciendo difícil distinguir los trabajadores subcontratados de los directamente contratados cuando desempeñan igual tarea o bien encontrándose en estas plantas tareas productivas que en su totalidad son realizadas por personal subcontratado.

\section{Clima Organizacional en empresas contratistas}

Snow $^{25}$ conceptualiza el clima organizacional como la percepción del empleado respecto al ambiente de trabajo y afirma que es uno de los factores de mayor influencia sobre el desempeño profesional. En este sentido, el clima organizacional es uno de los 
factores más influyentes sobre el nivel de satisfacción laboral de los trabajadores, concebido como un conjunto de propiedades medibles del ambiente de trabajo que son percibidos por las personas que trabajan en ella e influencian su motivación y comportamiento. Por tanto, el desempeño se encuentra determinado por la relación entre clima organizacional, bienestar de las personas en su trabajo y la calidad de vida laboral. ${ }^{26}$

Al clima organizacional se le entiende como un conjunto particular de prácticas y procedimientos organizacionales, tanto formales como informales. Es así como Cárdenas, Arciniegas \& Barrera ${ }^{27}$ definen el clima organizacional como un fenómeno que se traduce en un comportamiento que tiene consecuencias sobre la organización, tales como la productividad, satisfacción, rotación, entre otros. Es decir, que media entre los factores del sistema organizacional y las tendencias motivacionales de los trabajadores. La complejidad de estudiar los climas en las organizaciones ha llevado a dos niveles de estudio: el psicológico y el organizacional. ${ }^{28}$ El primero entendido a nivel individual, en tanto el segundo a nivel organizacional. Ambos aspectos son considerados fenómenos multidimensionales que describen la naturaleza de las percepciones que los empleados tienen de sus propias experiencias dentro de una organización. ${ }^{10}$

Además, el clima organizacional puede medirse con una diversidad de indicadores objetivos y subjetivos. Como se ha descrito anteriormente, este constructo como una característica objetiva de la organización, mientras que estudios más actuales la explican como un conjunto de opiniones, sentimientos y comportamientos que definen una organización. ${ }^{29}$ La percepción colectiva de los trabajadores acerca de su organización con respecto a una serie de dimensiones organizacionales es base del estudio de clima laboral. ${ }^{10,28}$

Así también, puede que existan múltiples climas dentro de la misma organización, ya que la vida en la organización puede variar en cuanto a las percepciones de los miembros según los niveles de la misma, sus diferentes lugares de trabajo, o las diversas unidades dentro del mismo centro de trabajo. ${ }^{10}$ El clima organizacional es abordado desde diversas perspectivas que van desde las características tanto del medio ambiente laboral (condiciones físicas del lugar de trabajo, tamaño de la organización, la estructura y las políticas de recursos humanos), hasta elementos como los valores y necesidades de los individuos, sus aptitudes, actitudes y motivación.

Aunque no es parte de alcance de este estudio, la satisfacción laboral está asociada al clima organizacional, ya que ambas dependen de las características de la organización y las condiciones de trabajo y, a su vez, son causa de múltiples formas de comportamiento organizacional. Sin embargo, los resultados de algunas investigaciones son contradictorios. Mientras en algunos estudios el clima organizacional se relaciona con la satisfacción laboral positivamente, los factores de riesgo psicosocial se relacionan negativamente con la satisfacción laboral; y el salario, las relaciones interpersonales, la organización del trabajo y las posibilidades de promoción son fuentes importantes de satisfacción laboral; otros estudios no parecen confirmar tales hallazgos. ${ }^{30} \mathrm{El}$ clima laboral y el outsourcing han sido estudiados por algunos autores que manifiestan que este tipo de contratación tiene el objetivo de concentrar los esfuerzos empresariales en el núcleo o especialización de la empresa, dejando en manos de empresas subcontratistas (incluyendo a sus empleados) todas las labores que no se consideren indispensables o cuya inclusión no agrega valor substancial a la cadena (las revisiones de la cadena de valor porterianas pueden verse como adeptas a esta conducta). Sin embargo, este modelo tiene serios contradictores que aducen que la clase de contrato a seleccionar no está sujeta a una dirección estratégica basada en funciones, sino que está soportada en la disminución de los costos. ${ }^{31}$

Cada organización va adoptando diversas condiciones y características que afectan directamente la manera de actuar de los integrantes, y la forma de percepción de dichas características que conforma el clima organizacional, por tanto el clima laboral puede ser estudiado de diversas maneras y es necesario comenzar desde el interior de éstas y conocer los factores que lo determinan. Se considera que se cuenta con un buen clima organizacional cuando los trabajadores desarrollan sus labores dentro en un medio ambiente interno favorable, usando sus conocimientos y habilidades de una manera conveniente y oportuna; al no ser así, usualmente nos encontramos con un empleado insatisfecho con su trabajo, aunque un mismo empleado puede estar satisfecho con algunos puntos en su trabajo e insatisfecho con otras áreas, haciendo imprescindible ubicar si existe una satisfacción completa o parcial. ${ }^{32}$

Aunque la literatura es escasa respecto a la medición del clima organizacional en el sector forestal, especialmente en sector producción, existen algunas evidencias como por ejemplo en México $^{33}$, donde se pudo establecer un estudio bajo el modelo de Litwin y Stringer, analizando cuatro dimensiones: estructura, relaciones, recompensa e identidad, que concluye que el contar con un "ejido" (porción de tierra de propiedad pública) ayuda a la motivación de los trabajadores.

\section{MÉTODO}

La propuesta sobre la percepción del clima organizacional se enmarca en un estudio de caso $^{34}$, cuyo objetivo es demostrar una parte de la realidad a analizar y no tiene la intención, a lo menos en esta etapa, de realizar inferencias hacia la población total. El diseño de esta fase de la investigación tiene un alcance exploratorio descriptivo, con aplicación de un instrumento cuantitativo que abordó la percepción de los trabajadores de faena de una empresa contratista del sector forestal-maderero de la Región del Bío-Bío, Chile. Es un estudio transversal, con un levantamiento de datos al término del año 2014.

En la empresa existe un total de 57 trabajadores, de los cuales el 91\% corresponde a trabajadores de faena y el 9\% restante a personal administrativo. Este primer segmento corresponde al grupo sujeto a estudio de esta investigación, dado que aunque se tuvo acceso al personal administrativo de la empresa, éste fue excluido dada que las características y condiciones higiénicas laborales son muy distintas a las de faena de terreno.

Por lo anterior, en definitiva la población está conformada por 52 trabajadores de faena de los cuales 49 contestaron el instrumento. Los trabajadores de faena se dividen en tres grupos: Cosecha (12 personas); Raleo A (18 personas) y Raleo B (19 personas). Las características de estos grupos son reflejadas en la Tabla 1.

\section{Instrumentos de recolección de datos}

Para realizar el levantamiento de información se utilizó el instrumento de clima organizacional diseñado por los autores Koys \& Decottis ${ }^{28}$ y adaptado por Chiang, Núñez, Martín \& Salazar ${ }^{32}$, el cual permitirá medir las variables de clima organizacional; se evalúa con una escala tipo Likert de cinco puntos donde 1 es Totalmente en desacuerdo y 5 es Muy de Acuerdo. 
Tabla 1.

Grupos de trabajo en faena.

\begin{tabular}{|c|c|c|c|c|}
\hline Grupo y Características & Sueldo & $\begin{array}{c}\text { Edad } \\
\text { promedic }\end{array}$ & $\begin{array}{l}\text { Experiencia } \\
\text { o promedio } \\
\text { (Años) }\end{array}$ & $\begin{array}{l}\text { Cantidad de } \\
\text { Trabajadores }\end{array}$ \\
\hline $\begin{array}{l}\text { Grupo COSECHA: Trabajadores con } \\
\text { más antigüedad en la empresa y por } \\
\text { ende con vasta experiencia laboral, su } \\
\text { remuneraciónes en base a producción, } \\
\text { en este grupo se concentran personas } \\
\text { que dominan a cabalidad las maqui- } \\
\text { narias dispuestas por la organización } \\
\text { y es por esto que se les designan } \\
\text { la última tecnología adquirida. }\end{array}$ & $\begin{array}{l}\text { Mayores } \\
\text { dentro } \\
\text { de la } \\
\text { empresa } \\
\text { (en base } \\
\text { a } \\
\text { producción). }\end{array}$ & 52 & 15 & 12 \\
\hline $\begin{array}{l}\text { Grupo RALEO A: Se compone por } \\
\text { trabajadores jóvenes que aún están en } \\
\text { etapa de aprendizaje y trabajadores de } \\
\text { mediana experiencia, no obstante } \\
\text { conocen la maquinaria de la empresa } \\
\text { y a medida que pasa el tiempo se van } \\
\text { consolidando dentro de ella. }\end{array}$ & $\begin{array}{l}\text { Nivel } \\
\text { promedio } \\
\text { de la } \\
\text { empresa } \\
\text { (en base } \\
\text { a } \\
\text { producción). }\end{array}$ & 25 & 5 & 18 \\
\hline \multirow[t]{2}{*}{$\begin{array}{l}\text { Grupo RALEO B: Se compone por } \\
\text { trabajadores recién egresados de } \\
\text { liceos técnicos rurales cercanos a las } \\
\text { faenas, están en proceso de aprendi- } \\
\text { zaje y en constante supervisión de } \\
\text { los jefes de faena. }\end{array}$} & $\begin{array}{l}\text { Nivel } \\
\text { menor al } \\
\text { promedio } \\
\text { dentro } \\
\text { de la } \\
\text { empresa. }\end{array}$ & 22 & 3 & 19 \\
\hline & & & Total & 49 \\
\hline
\end{tabular}

Fuente: Elaboración propia

Seis elementos expresados en las preguntas se encuentran de forma negativa y son valorados de forma inversa. En base a los estudios de Chiang et al y Koys \& Decottis ${ }^{32,28}$, se proponen ocho escalas de clima organizacional: autonomía, cohesión, confianza, presión, apoyo, reconocimiento, equidad e innovación.

La participación de los trabajadores fue voluntaria y anónima. El cuestionario fue autoaplicado y sin control de tiempo. La encuesta contaba con una explicación de la investigación en la primera página y el trabajo de campo fue realizado por una persona externa a la empresa con el objetivo de mantener el anonimato.

\section{Técnicas de análisis de los datos}

Para analizar las respuestas de los participantes en la encuesta, se utilizó el programa estadístico Statistical Package for the Social Sciences (SPSS) versión 18, siendo la herramienta de apoyo estadístico en el estudio. ${ }^{35}$

Se realizó un análisis de fiabilidad según el Alpha de Cronbach a todo el cuestionario y además este análisis se efectuó considerando cada grupo de encuestados. Asimismo, para cada una de las dimensiones del instrumento se calculó la media y la desviación estándar, considerando tanto el total de sujetos a estudio como según grupo de trabajo al que pertenecen.

\section{RESULTADOS}

Se determinó el indicador Alpha de Cronbach para la totalidad del instrumento de diagnóstico (40 elementos), donde el resultado es 0,801 (Ver Tabla 2), considerado altamente aceptable bajo los criterios de George \& Mallery. ${ }^{35}$ Ahora bien, para los tres grupos encuestados los resultados fueron aceptables (Ver Tabla 3). De manera especial, hay que interpretar detenidamente el valor del grupo Raleo B, ya que se obtuvo un resultado de 0,662 .
Tabla 2.

Estadísticos de fiabilidad.

$\begin{array}{lc}\text { Alfa de Cronbach } & N^{\circ} \text { de elementos } \\ 0,801 & 40\end{array}$

Tabla 3.

Estadísticos de fiabilidad por grupo.

$\begin{array}{lcc}\text { Grupo encuestado } & \text { Alfa de Cronbach } & N^{\circ} \text { de trabajadores } \\ \text { Cosecha } & 0,890 & 12 \\ \text { Raleo A } & 0,799 & 18 \\ \text { Raleo B } & 0,662 & 19\end{array}$

La encuesta está diseñada por 40 preguntas; con ella se recoge la percepción del clima organizacional de 49 trabajadores de faena con el objetivo de evidenciar el estado de esta variable, la cual es evaluada por la empresa principal dentro de un conjunto de otras variables que son parte del estándar medido en la certificación de manejo forestal sostenible.

Entre los primeros hallazgos que permiten caracterizar el grupo estudio, se puede observar que existe una heterogeneidad entre los tres grupos de trabajo de faena; el de cosecha que tiene mayor tiempo en la empresa, mayor experiencia y con mejores condiciones remunerativas. En tanto los dos grupos de raleo (A y B) tienen poca experiencia en relación a los primeros, menos edad, condiciones remunerativas más desmejoradas e indexada a productividad y, además, existe una mayor rotación de trabajadores en el sistema.

\section{Valoración de las percepciones}

Las valoraciones de los trabajadores sobre el clima organizacional han sido sobre la media en general para todas las dimensiones. Encontrando una media general de 3,86 en una escala de 1 al 5, con una desviación típica de 0,92 para toda la empresa y que se desglosa según se observa en la Tabla 4:

Tabla 4.

Estadisticos de fiabilidad por grupo.

\begin{tabular}{|c|c|c|c|c|c|c|c|c|}
\hline Dime & $\begin{array}{r}\text { Total } \\
\text { Media }\end{array}$ & $\begin{array}{l}\text { npresa } \\
\text { esv. Típ }\end{array}$ & $\begin{array}{l}\text { Gru } \\
\text { Med }\end{array}$ & $\begin{array}{l}\text { secha } \\
\text { sv. Típ }\end{array}$ & $\begin{array}{l}\text { Gru } \\
\text { Med }\end{array}$ & $\begin{array}{l}\text { aleo A } \\
\text { esv. Típ }\end{array}$ & $\begin{array}{l}\text { Gru } \\
\mathrm{Med}\end{array}$ & $\begin{array}{l}\text { aleo B } \\
\text { esv. Típ }\end{array}$ \\
\hline Autonomía & 4,13 & 0,88 & 4,18 & 0,86 & 4,14 & 0,89 & 4,13 & 0,87 \\
\hline Thesión & 4,05 & 0,92 & $J_{1}$ & 0,92 & 4,20 & 0,90 & 3,98 & 0,95 \\
\hline & 4,1 & 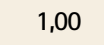 & & 1,01 & & 1,02 & & 1,00 \\
\hline . & 3,45 & 08 & & 04 & 3 & 1,13 & & 1,09 \\
\hline r & & & & & & 0 & & 0,84 \\
\hline leconocimiento & 3,53 & 0,00 & & , & & 0,85 & & 0,85 \\
\hline Equidad & 3,7 & 0,96 & & 0,98 & 3,8 & 0,95 & 3,77 & 1,00 \\
\hline Innovación & 3,77 & 0,90 & 3,7 & 0,90 & 3,8 & 0,88 & 3,74 & 0,95 \\
\hline romedio general & al 3,86 & 0,92 & 3,85 & 0,93 & 3,91 & 0,93 & 3,84 & 0,94 \\
\hline
\end{tabular}

\section{DISCUSIÓN}

Si se analiza con mayor detalle las dimensiones -en base a los conceptos de Koys \& Decottis ${ }^{28}$ que obtuvieron mayor puntaje, destacan a nivel de empresa la "autonomía" $(4,13)$, la cual tiene que ver con la autodeterminación y responsabilidad necesaria en la toma de decisiones con respecto a procedimientos del trabajo, metas y prioridades de la mayor parte de los trabajadores. La segunda dimen- 
sión es la "confianza" $(4,11)$, respecto a la percepción de la libertad para comunicarse abiertamente con los superiores, para tratar temas sensibles o personales con la confidencia suficiente de que esa comunicación no será violada o usada en contra de los miembros, aunque fue una de las que obtuvo mayor desviación típica $(1,0)$ en virtud de la dispersión entre los grupos. La tercera con más alto puntaje es el "apoyo" $(4,08)$; esta tiene que ver con la percepción que tienen los miembros acerca del respaldo y tolerancia en el comportamiento dentro de la institución, lo que incluye el aprendizaje de los errores por parte del trabajador, sin miedo a la represalia de sus superiores o compañeros de trabajo.

Respecto a las dimensiones que obtuvieron menor puntaje, se puede mencionar la "presión" $(3,45)$ referida a la percepción que existe con respecto a los estándares de desempeño, funcionamiento y finalización de la tarea. Existe cierta preocupación por esta perspectiva que tiene que ver con las exigencias en los sistemas de turno y las presiones en relación a las diferentes formas de pago de remuneraciones, algunos en base a lo que producen y otros en forma fija. Asimismo, es una de las dimensiones con mayor desviación típica $(1,08)$. Una segunda dimensión menos valorada es el "reconocimiento" $(3,53)$, que es la percepción que tienen los miembros de la organización con respecto a la recompensa que reciben, por su contribución a la empresa, es decir, se percibe de poca claridad por parte de los jefes respecto a los reconocimientos hacia sus colaboradores. Una tercera dimensión con menor puntaje es la "innovación" $(3,77)$, es decir, la percepción acerca del ánimo que se tiene para asumir riesgos, ser creativo y asumir nuevas áreas de trabajo, aunque tenga poco o nada de experiencia.

$\mathrm{Al}$ realizar un análisis transversal de las percepciones entre los distintos grupos, el grupo "Raleo A" obtuvo la mayor valoración $(3,91)$, luego el grupo "Cosecha" $(3,85)$ y con menor valoración el grupo "Raleo B" $(3,84)$, siendo este último donde se observa la mayor dispersión con una desviación típica $(0,94)$, atribuibles a la heterogeneidad y baja experiencia en el sector del grupo. En este mismo ámbito, la mayor valoración se encuentra en la dimensión de "cohesión" del Grupo "Raleo A" $(4,20)$, donde se destaca la percepción de las relaciones entre los trabajadores dentro de la organización, la existencia de una atmósfera amigable y de confianza y proporción de ayuda material en la realización de las tareas, donde se observa trabajo en equipo ya que los trabajadores se sienten parte del grupo, lo que se puede deber a que sienten que poseen cosas en común con sus compañeros. Otra dimensión destacada es la "autonomía” $(4,18)$ en el grupo de "Cosecha".

Asimismo, la menor valoración es la dimensión "presión" en los grupos "Raleo A" $(3,40)$ y "Raleo B" $(3,47)$, donde se observa las altas exigencias que tienen estos trabajadores asociadas a las metas de producción que se encuentran indexadas a sueldo. Otra dimensión con baja valoración es el "reconocimiento" en el grupo de "Cosecha" $(3,47)$, asociada a la ausencia de políticas de recursos humanos para con los trabajadores.

\section{CONCLUSIONES}

La elección de las empresas contratistas en el sector forestal es cada vez es más exigente, en base a criterios económicos, técnicos y sociales. Contar con trabajadores altamente preparados, motivados y comprometidos para las empresas contratistas es un desafío permanente; por ello, la realización de la encuesta de clima es fundamental para saber el estado de la organización, pudiendo identificar sus fortalezas y debilidades, y sabiendo que un clima laboral es un tema de preocupación día a día. La encuesta no es un objetivo en sí misma, hay que entenderlo como un instrumento más que ayuda a medir las percepciones de los trabajadores, entregando importantes insumos que permitan generar acciones de intervención en aquellos aspectos más débiles que impactan su desempeño, mejorando las prácticas de trabajo, manteniendo un ambiente laboral propicio para los trabajadores $\mathrm{y}$, especialmente, que estos últimos desarrollen sus funciones en condiciones adecuadas.

En este contexto, el haber aplicado una encuesta de clima organizacional para la empresa constituye un diagnóstico externo sobre el estado de clima entre sus trabajadores, quienes en un 88\% respondieron la encuesta de cuarenta preguntas, siendo altamente representativo y con una fiabilidad del "alfa de Cronbach" del 80,5. Teniendo como premisa que el instrumento mide percepciones, el estudio de caso arrojó que la empresa tiene un buen clima laboral, en las 34 preguntas positivas se obtuvo un promedio de 30,7\% de la mayor puntuación (muy de acuerdo) y sumado la respuesta "de acuerdo"; esta alcanza en forma conjunta 61,9\% de las respuestas, lo cual refleja una buena percepción de clima.

Asimismo, se refleja que ninguna pregunta superó el 14\% de negatividad. Sin embargo, existe un alto porcentaje de respuestas "no estoy seguro", las cuales pueden indicar motivos diferentes, como por ejemplo: indiferencia al instrumento, inhibición a responder negativamente, forma de ocultar su verdadera respuesta, entre otros. Probablemente debido a estas respuestas es que en evaluaciones anteriores aplicadas por la empresa mandante haya bajado el indicador de clima organizacional. Al tratar de explicar la causa de este tipo de respuesta también se puede inferir la alta rotación entre faenas, la gran diferencia entre la edad promedio de los grupos, experiencia laboral, condiciones ambientales de trabajo, las actividades que desarrollan y antigüedad en la empresa.

Lo anterior abre un camino para trabajar en el porcentaje de respuestas No estoy seguro, donde los trabajadores no tienen claros ciertos aspectos dentro de la organización y son más susceptibles a recibir mejoras y transformarlas en percepciones positivas dentro de la empresa.

El instrumento aplicado presenta una alta confiabilidad estadística, lo cual potencia los resultados obtenidos. Sin embargo, el estudio debe entenderse en el contexto en el cual fue aplicado, es decir, en un sector industrial donde las empresas contratistas están fuertemente subordinadas a los lineamientos de las grandes empresas forestales, con bajo poder de negociación. Por consiguiente, los criterios de eficiencia y reducción de costos están presentes en la relación comercial entre los actores, en los procesos de licitación, lo cual puede llevar a las empresas contratistas a un control exhaustivo de su estructura de costos que, de una u otra forma, impacta en los sueldos y compensaciones de sus trabajadores y, a su vez, repercute en la estabilidad, en el nivel de rotación de trabajadores y, por supuesto, en el clima organizacional, objeto central de nuestro estudio. Y bien se sabe, de acuerdo a la literatura, que un buen clima no se construye en el corto plazo, sino más bien en un proceso complejo que se perfecciona a lo largo del tiempo.

Otro aspecto, y tal como se ha expresado, es que la investigación propuesta mide percepciones, desde ocho perspectivas del clima organizacional existente, y en una empresa del tamaño analizado es inminentemente posible que, aunque se cautele el anonimato de los encuestados, no exista la confianza tal para expresar su real opinión ante el temor de posibles consecuencias en su situación contractual con la empresa. Esto ha llevado a que los resultados obtenidos en la 
investigación se entreguen en forma general, no permitiendo identificar resultados separados por cada grupo.

Lo que es evidente es que las buenas prácticas, los sistemas de manejo sostenible cobran sentido, más aun con los procesos de certificación y contribuyen a crear un ambiente acorde entre las empresas demandantes y contratistas. Es un hecho que las condiciones laborales de los trabajadores tercerizados son distintas a la de las empresas mandantes y existe un imperativo de ir mejorando su situación, ya que ésta incide en el desempeño general de todo el sistema. Muchos trabajos coinciden en la importancia de tener un buen clima organizacional colocando como eje central el bienestar de sus trabajadores. Este trabajo constituye un avance al tratar de adaptar un modelo de medición ${ }^{32}$ que se ha realizado en otros sectores a uno con características muy especiales, donde las condiciones ambientales muchas veces son adversas, donde a pesar de los avances se indica que aún persisten grados de precariedad, y donde las relaciones laborales se sustentan esencialmente en la estructura de costos y bien sabemos que la subcontratación y la flexibilización laboral que se genera en este sector inhibe en gran parte la posibilidad de sindicalización y, por ende, saber claramente cuál es el clima organizacional existente en las empresas del sector.

Por último, en futuras investigaciones es preciso perfeccionar la información sobre la calidad del empleo en el sector si se persigue mejorar las condiciones laborales. Al igual que en otras dimensiones que son certificadas en el manejo forestal sostenible respecto al recurso humano, como por ejemplo la tasa de accidentabilidad analizada por Ackerknecht ${ }^{5}$, el clima organizacional constituye un factor más que ayuda a la generación de espacios de trabajo seguros $\mathrm{y}$ saludables en las operaciones forestales y madereras, siendo un elemento que agrega valor al sector.

\section{Agradecimientos}

Se agradece el aporte al trabajo empírico de las ingenieras Srtas. Cristina Salazar y Silvana Huichahuala.

\section{REFERENCIAS}

1. Grosse $\mathrm{H}, \mathrm{Barros} \mathrm{S}$. Los resultados de una politica estatal estable de fomento forestal. El caso chileno. Ciencia e Investigación Forestal- INFOR Chile. 2013;19(1):73-98.

2. Nazif I. Plantaciones y Pobreza en Comunas Forestales. Forestación y Estilo de Desarrollo. Santiago: CONAF; 2014. (Documento Técnico, 216).

3. Andersson K, Lawrence D, Zavaleta J, Guariguata MR. More Trees, More Poverty? The Socioeconomic Effects of Tree Plantations in Chile, 2001-2011. Environ Manage. 2016;57(1):123-136.

4. Chile. INFOR. Datos forestales 2014 [en línea]. Santiago: INFOR; 2014 [consultado nov 2015]. Disponible en: http://www. infor.cl/

5. Ackerknecht C. Occupational Accidents Footprint : New Concept Linked to Chain of Custody in Sustainable Forest Management. Cienc Trab. 2014;51:131-136.

6. Alves R, Gonçalves L, Basso V, Lopes-da-Silva EM. Plantações florestais e a proteção de florestas nativas em unidades de manejo certificadas no Brasil. Rev Arvore. 2011;35(4):859-866.

7. Sistema Chileno de Certificación de Manejo Forestal Sustentable-CERTFOR. Estándar Certfor de Manejo Forestal Sustentable para Plantaciones (DN-02-05) [en línea]. Santiago: CERTFOR-PEFC; 2013 [consultado nov.2015].Disponible en: http://www.certfor.org/imgmodulo/archivos1/25.pdf

8. Ismail M, Rossi A, Geiger N. A Compilation of Bioenergy Sustainability Initiatives: Update. FSC Principles and Criteria for Forest Stewardship [en línea]. Rome: BEFSCI-FAO; 2011 [consultado nov. 2015]. Disponible en: http://www. fao.org/bioenergy/28178-0113cfa7c8acba716901b6cefedfe030.pdf

9. Verjans JM. Interpretación de los principios y criterios del FSC en manejo forestal. Rev For Mesoam Kurú. 2011;8(21):13-16.

10. Chiang $M$, Salazar $M$, Huerta $P$, Nuñez A. Clima organizacional y satisfacción laboral en organizaciones del sector estatal (Instituciones públicas). Desarrollo, adaptación y validación de instrumentos. Universum. 2008;23(2):66-85.

11. Falabella G, Gatica F. Sector forestal-celulosa, agricultura de secano e industria en el Gran Concepción: ¿encadenamiento productivo o enclave? Rev Cepal. 2014;112:197-214.

12. PROGEA-UNTEC. Aporte Económico y Social Del Sector Forestal en Chile y Análisis de Encadenamientos; Resumen Ejecutivo 2014 para CORMA [en línea]. Santiago: Universidad de Chile; 2014 [consultado nov. 2015]. Disponible en: http://www.corma.cl/_file/material/resumen-ejecutivo-evaluacion-del-aporteeconomico-y-social-del-sector-forestal-2014.pdf

13. Donoso PJ, Otero L. Hacia una definición de pais forestal: ¿Dónde se sitúa Chile? Bosque. 2005;26(3):5-18.

14. Canals M. Los Trabajadores Forestales de la VIII Región. Condiciones de Vida y Horizontes Políticos 1990-2013. Santiago: Universidad de Chile; 2014.

15. Toppinen A, Virtanen A, Mayer A, Tuppura A. Standardizing Social Responsibility via ISO 26000: Empirical Insights from the Forest Industry. Sustain Dev. 2015; 23(3):153-166.

16. Organización Internacional del Trabajo-OIT. El Trabajo decente en la Industria Forestal Chilena [en línea]. Santiago: OIT; 2012 [consultado dic 2015].
Disponible en: https:\|www.oitchile.cl.

17. Muthoo MK. La certificación forestal y la economía verde. Unasylva. 2012;63(1):17-23.

18. Li N, Toppinen A. Corporate responsibility and sustainable competitive advantage in forest-based industry: Complementary or conflicting goals? Forest Policy Econ. 2011;13(2):113-123.

19. Ackerknecht C. Occupational Safety Indicators for Forest Operations, Sawmilling and Wood-Based Panels Manufacture ; an International Benchmarking. Cienc Trab. 2015;17(53):89-98.

20. Ganga F. Externalización de funciones: algunas reflexiones teóricas. Estudios Gerenciales. 2008;24(107):107-135.

21. Rojas I. La externalización laboral y la cesión ilegal de trabajadores en el sistema jurídico chileno.lus et praxis. 2010;16(1):171-196.

22. Fernández $E_{1}$ Piñeiro D. Tercerización laboral en el Uruguay: estudio comparado de contratistas de trabajo y de maquinaria en el medio rural. Agrociencia Uruguay. 2013;17(2):141-149.

23. Echeverria M. La Historia inconclusa de la subcontratación y el relato de los Trabajadores. Santiago: Dirección del Trabajo, Gobierno de Chile; 2010.

24. Ávila A, Ribeiro G. A precarização do Trabalho e a Produção de acidentes na colheita de árvores. Caderno CHR. 2011;24(62):385-396.

25. Snow J. Enhancing Work Climate to Improve Performance and Retain Valued Employees. J Nurs Adm. 2002;32(7-8):393-397.

26. Manosalvas CA, Manosalvas LO, Nieves J. El clima organizacional y la satisfacción laboral: un análisis cuantitativo riguroso de su relación. AD-minister. 2015;26:5-15.

27. Cárdenas $L$, Arciniegas $Y$, Barrera M. Modelo de intervención en clima organizacional. Int J Psychol Res. 2009;2(2):121-127.

28. Koys DJ, Decottis TA. Inductive Measures of Psychological Climate. Hum Relat. 1991;44(3):265-285.

29. Rahimic Z. Influence of Organizational Climate on Job Satisfaction in Bosnia and Herzegovina Companies. Int Bus Res. 2013;6(3):129-139.

30. Arias $W$, Arias $G$. Relación entre el clima organizacional y la satisfacción laboral en una pequeña empresa del sector privado. Cienc Trab. 2014;16(51):185-191.

31. Juliao $D$, Sánchez $C$, Martínez D. Diferencias del clima laboral entre empleados contratados por outsourcing y empleados directos : evidencia empirica de la zona norte de Colombia. Universidad \& Empresa. 2014;16(26):277-295.

32. Chiang M, Núñez A, Martín M, Salazar M. Compromiso del Trabajador hacia su Organización y la relación con el Clima Organizacional: Un Análisis de Género y Edad. Panorama Socioeconómico. 2010;28(40):92-103.

33. Geovani E, Arrieta $D$, Moreno $H$, González M, Monsisvais M. La percepción del clima organizacional en el personal de producción de un ejido forestal en México. RGN. 2013;1(2):81-89.

34. Stake RE. Investigación con estudio de casos. Madrid: Edcs. Morata; 1998.

35. George D, Mallery P. SPSS for Windows Step by Step: A Simple Guide and Reference. 11.0 Update. 4th ed. Boston: Allyn \& Bacon; 2003. 